\title{
Investigating ion recombination effects in a liquid-filled ionization chamber array used for IMRT QA measurements
}

\author{
Cory Knill, ${ }^{a)}$ Michael Snyder, and Joseph T. Rakowski \\ Department of Radiation Oncology, Karmanos Cancer Institute, Detroit, Michigan 48201 and Department \\ of Radiation Oncology, Wayne State University School of Medicine, Detroit, Michigan 48201 \\ Ling Zhuang \\ Department of Radiation Oncology, Wayne State University School of Medicine, Detroit, Michigan 48201 \\ Martha Matuszak \\ Department of Radiation Oncology, University of Michigan Health System, Ann Arbor, Michigan 48109 \\ Jay Burmeister \\ Department of Radiation Oncology, Karmanos Cancer Institute, Detroit, Michigan 48201 and Department \\ of Radiation Oncology, Wayne State University School of Medicine, Detroit, Michigan 48201
}

(Received 13 August 2015; revised 17 March 2016; accepted for publication 3 April 2016; published 22 April 2016)

Purpose: PTW's Octavius 1000 SRS array performs IMRT quality assurance (QA) measurements with liquid-filled ionization chambers (LICs) to allow closer detector spacing and higher resolution, compared to air-filled QA devices. However, reduced ion mobility in LICs relative to air leads to increased ion recombination effects and reduced collection efficiencies that are dependent on Linac pulse frequency and pulse dose. These pulse parameters are variable during an IMRT delivery, which affects QA results. In this study, (1) 1000 SRS collection efficiencies were measured as a function of pulse frequency and pulse dose, (2) two methods were developed to correct changes in collection efficiencies during IMRT QA measurements, and the effects of these corrections on QA pass rates were compared.

Methods: To obtain collection efficiencies, the OCTAVIUS 1000 SRS was used to measure open fields of varying pulse frequency, pulse dose, and beam energy with results normalized to air-filled chamber measurements. Changes in ratios of 1000 SRS to chamber measured dose were attributed to changing collection efficiencies, which were then correlated to pulse parameters using regression analysis. The usefulness of the derived corrections was then evaluated using $6 \mathrm{MV}$ and 10FFF SBRT RapidArc plans delivered to the OCTAVIUS 4D system using a TrueBeam (Varian Medical Systems) linear accelerator equipped with a high definition multileaf collimator. For the first correction, MATLAB software was developed that calculates pulse frequency and pulse dose for each detector, using measurement and DICOM RT Plan files. Pulse information is converted to collection efficiency, and measurements are corrected by multiplying detector dose by ratios of calibration to measured collection efficiencies. For the second correction the MU/min in the daily 1000 SRS calibration was chosen to match the average MU/min of the volumetric modulated arc therapy plan. Effects of the two corrections on QA results were examined by performing 3D gamma analysis comparing predicted to measured dose, with and without corrections.

Results: Collection efficiencies correlated linearly to pulse dose, while correlations with pulse frequency were less defined, generally increasing as pulse frequency decreased. After complex MATLAB corrections, average 3D gamma pass rates improved by $[0.07 \%, 0.40 \%, 1.17 \%]$ for $6 \mathrm{MV}$ and $[0.29 \%, 1.40 \%, 4.57 \%]$ for $10 \mathrm{FFF}$ using $[3 \% / 3 \mathrm{~mm}, 2 \% / 2 \mathrm{~mm}, 1 \% / 1 \mathrm{~mm}]$ criteria. Maximum changes in gamma pass rates were $[0.43 \%, 1.63 \%, 3.05 \%]$ for $6 \mathrm{MV}$ and $[1.00 \%, 4.80 \%, 11.2 \%]$ for $10 \mathrm{FFF}$ using $[3 \% / 3 \mathrm{~mm}, 2 \% / 2 \mathrm{~mm}, 1 \% / 1 \mathrm{~mm}]$ criteria. On average, pass rates of simple daily calibration corrections were within $1 \%$ of complex MATLAB corrections.

Conclusions: OCTAVIUS 1000 SRS ion recombination effects have little effect on $6 \mathrm{MV}$ measurements. However, the effect could potentially be clinically significant for higher pulse dose unflattened beams when using tighter gamma tolerances, especially when small aperture sizes are used, as is common for SRS/SBRT. In addition, ion recombination effects are strongly correlated to changing $\mathrm{MU} / \mathrm{min}$, therefore MU/min used in daily 1000 SRS calibrations should be matched to the expected average MU/min of the IMRT plan. (C) 2016 American Association of Physicists in Medicine. [http://dx.doi.org/10.1118/1.4946822]

Key words: 1000 SRS, ion recombination, Octavius 4D 


\section{INTRODUCTION}

IMRT quality assurance (QA) consists of delivering patient plans to dosimetric measuring devices and comparing the dose to that predicted by the treatment planning system (TPS). The devices must accurately measure dose with high enough resolution to sufficiently characterize the field. When small field sizes are used this requires the close placement of small detectors. ${ }^{1,2}$ This can cause issues when using air-filled ionization chambers, typically used in nonIMRT radiation dose measurements. ${ }^{3,4}$ The low-density air results in less scatter and a low signal to noise ratio, which limits the proximity and size of the detectors.

One potential solution is to use liquid-filled ionization chambers (LICs), which use higher-density liquid to increase response and scatter. ${ }^{5,6}$ However, there is a tradeoff- the liquid reduces ion mobility, leading to ion collection times that are approximately 100 times larger than air-filled chambers, which increases ion recombination effects. ${ }^{7-13}$ Ion recombination effects have been studied in LICs and found to be proportional to the frequency and dose of the pulses delivered to the detector, among other parameters that are typically constant during a measurement. The dose and frequency of the pulses at each detector will vary during an IMRT delivery due to the changing multileaf collimator (MLC) aperture and changing machine dose rate $(\mathrm{MU} / \mathrm{min})$, respectively. This could potentially introduce ion recombination effects into QA measurements, resulting in a distortion of the dose map and overall IMRT QA results.

The PTW 1000 SRS array (PTW, Freiburg, Germany) implements the above described LICs, for small field IMRT measurements. ${ }^{14}$ The goal of this research is to determine the effect of ion recombination on clinical 1000 SRS volumetric modulated arc therapy (VMAT) QA measurements and investigate two potential ion recombination correction methods.

\section{MATERIALS AND METHODS}

The device used in this study is the 1000 SRS detector. The 1000 SRS detector consists of 977 isooctane-filled LICs. The chambers have dimensions $2.3 \times 2.3 \times 0.5 \mathrm{~mm}$ (depth). The chambers are arranged in a planar array with a spacing of $2.5 \mathrm{~mm}$ in the center of the array and $5.0 \mathrm{~mm}$ at the periphery (Fig. 1). During measurements the array was placed in a rotating Octavius 4D phantom, which uses an inclinometer to keep the array aligned perpendicular to the axis of the beam. ${ }^{15}$ All measurements were performed on a TrueBeam linear accelerator (Varian Medical Systems, Pal Alto, CA) equipped with a high definition multileaf collimator (HD-MLC).

\section{A. LIC ion recombination}

The evaluation of the 1000 SRS detector began by determining the correlation between collection efficiency and pulse dose for the 1000 SRS LICs in $6 \mathrm{MV}$ and 10FFF beams. This investigation consisted of exposing the 1000 SRS detector to different pulse dose rates, measuring the dose and comparing the results to true dose measured with a $0.125 \mathrm{~cm}^{2}$

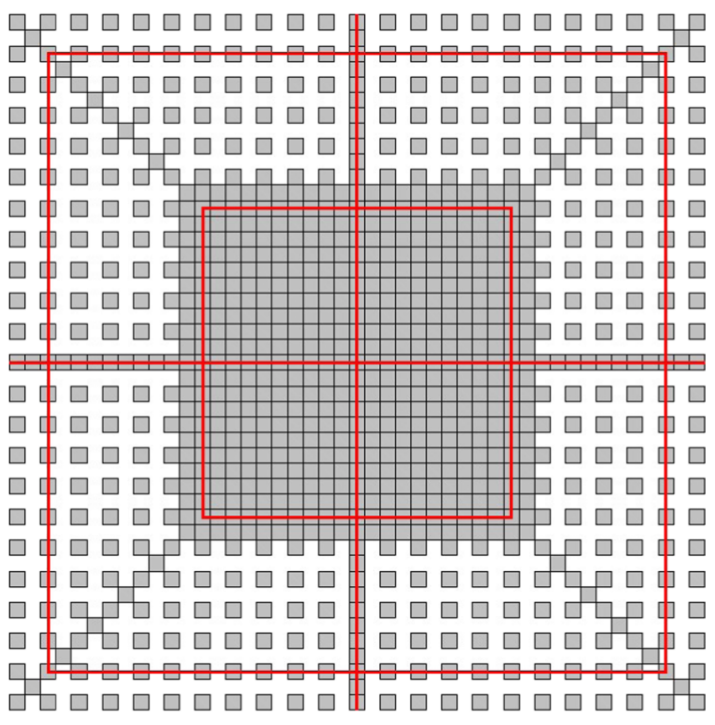

FIG. 1. 1000 SRS detector locations. Detectors are $2.3 \mathrm{~mm}$ wide squares. Centroid spacing is $2.5 \mathrm{~mm}$ in the center and $5.0 \mathrm{~mm}$ in the peripheral.

model 31010 air-filled ionization chamber (PTW, Freiburg, Germany) to determine the LIC collection efficiencies.

To create a consistent setup where the 1000 SRS detector measured the same pulse dose as the ion chamber, the detectors were alternately placed at the same location in a solid water stack. Prior to measurements, both devices were preirradiated, per manufacturer recommendations. After preirradiation, $9.1 \mathrm{~cm}$ of solid water was placed on top of the 1000 SRScombined with the $0.9 \mathrm{~cm}$ buildup in the device this resulted in an equivalent depth of $10 \mathrm{~cm}$. The distance to the surface of the solid water, or source-to-phantom distance (SPD), was set to $100 \mathrm{~cm}$. Using a $10 \times 10 \mathrm{~cm}$ field, 100 MUs were delivered to the 1000 SRS with the maximum dose rate of 600 and $2400 \mathrm{MU} / \mathrm{min}$ for $6 \mathrm{MV}$ and 10FFF, respectively. For subsequent measurements, the SPD and buildup on top of the 1000 SRS were varied to produce different pulse doses at the detectors. For each SPD and depth combination, the 1000 SRS dose measurement was divided by the ion chamber charge reading, which was corrected for $P_{\mathrm{tp}}, P_{\mathrm{pol}}$, and $P_{\text {ion }}$ using TG51 formalisms. ${ }^{16}$ These ratios were subsequently normalized to the dose/charge ratio at $100 \mathrm{~cm}$ SPD and $10 \mathrm{~cm}$ depth. The change in the dose/charge ratio at each SPD/depth combination was attributed to the different collection efficiencies of the LICs caused by the different pulse doses.

To correlate the LIC collection efficiencies with pulse dose, the pulse dose at the 1000 SRS central chamber was calculated for each SPD. The dose and number of pulses were determined separately and then used to calculate pulse dose. First, dose was obtained for $100 \mathrm{~cm}$ SPD by simulating the delivery in the Eclipse TPS (Varian Medical Systems, Pal Alto, CA) and calculating dose to the center detector. To eliminate any potential dosimetric effects caused by calculating dose in the treatment planning system at different SPDs, the ratios of the corrected ionization chamber readings were used to convert dose at $100 \mathrm{~cm}$ SPD to dose at other SPDs.

The number of pulses was determined from the pulse repetition frequency of the Linac and the delivery time. The 
deliveries were performed at the maximum $\mathrm{MU} / \mathrm{min}(600$ and $2400 \mathrm{MU} / \mathrm{min}$ for $6 \mathrm{X}$ and 10FFF, respectively), which corresponds to a Linac pulse frequency of $360 \mathrm{~Hz}$. Pulse frequency and calculated dose to the 1000 SRS detector were used to calculate pulse dose at each SPD.

The collection efficiencies were plotted against the pulse dose for different SPDs to obtain the pulse dose dependency of the 1000 SRS detector central chamber. The plotted data were modeled using a linear least-square fitting method. Based on the fitting results, a constant was subtracted from all of the collection efficiency data so that the modelled collection efficiency was 1.0 for a $0.0 \mathrm{mGy} /$ pulse rate.

To study the effect of pulse frequency on collection efficiency, the above procedure was repeated with different $\mathrm{MU} / \mathrm{min}$. The set $\mathrm{MU} / \mathrm{min}$ values were used to calculate average pulse frequency using the linear correlation between the two parameters and the fact that the max MU/min corresponded to a $360 \mathrm{~Hz}$ pulse frequency.

\section{B. IMRT ion recombination corrections}

The following is a brief description of how the 1000 SRS is used in the Octavius 4D system for clinical IMRT QA measurements. 1000 SRS measurements are performed in the Octavius 4D system, which consists of a rotating phantom that keeps the array perpendicular to the beam using an inclinometer attached to the gantry. Prior to a QA measurement, the central chamber of the 1000 SRS is calibrated to a known dose using a reference beam from the Linac. A default Co-60 array calibration file, included with the device, has chamber-specific calibration factors, which are scaled using the daily central chamber calibration, thereby calibrating the whole array. Once calibrated, treatment fields are delivered to the 1000 SRS, which measures dose in user defined collection intervals $(0.2 \mathrm{~s}$ for this study) as a function of gantry angle. For each collection interval, a vendor-supplied analysis software (VeriSoft) takes the measured dose and uses PDDs to back-project a 3D dose throughout the Octavius 4D phantom. The total dose, from all collection intervals, is combined and compared to the TPS using a 3D gamma analysis. ${ }^{17,18}$

The daily 1000 SRS calibration is performed at a department specific standard pulse frequency and pulse dose. During a 1000 SRS measurement, the changing MU/min and MLC aperture will cause the pulse frequency and dose to vary across the detector, which will cause changes in the collection efficiency. The changing collection efficiency could potentially cause errors in the IMRT QA measurement. To determine the magnitude of this effect the collection efficiencies determined in Subsection 2.A were used to correct clinical 1000 SRS measurements. The details of the IMRT measurements, the corrections, and the IMRT analysis are presented in Subsections 2.C-2.E.

\section{C. VMAT measurements}

The effects of ion recombination were studied in seven clinical SBRT plans: four lung, two spine, and one liver plan. The number of available clinical lung SBRT cases far outweighed spine and liver cases; however, a variety of plans were chosen to provide a broad case-set for studying ion recombination effects. All plans used RapidArc deliveries. All of the plans were replanned on the same machine (TrueBeam) for both $6 \mathrm{MV}$ and 10FFF energies, using the original clinical dose objectives specified by the physician and a $1.5 \mathrm{~mm}$ calculation voxel size, per department protocol. Each plan consisted of 4 or 6 arcs. In total, 34 arcs were measured for each energy. Each arc was delivered individually to the Octavius 4D. The measured dose was compared to the dose calculated in the TPS using a 3D gamma analysis. In this work the 3D gamma analysis was performed with a $3 \% / 3 \mathrm{~mm}, 2 \% / 2 \mathrm{~mm}$, and $1 \% / 1 \mathrm{~mm}$ global criteria using a threshold that excluded measurements below $10 \%$ of the maximum calculated TPS dose. Finally, the measurements were corrected for ion recombination effects using the procedures in Sec. 2.D, and then reanalyzed.

\section{D. Measurement corrections}

Ion recombination effects in 1000 SRS measurements are caused by the difference in collection efficiencies between the daily array calibration and the IMRT delivery. To correct this effect in the 1000 SRS measurements, the pulse frequencies and pulse dose for both calibration and the IMRT measurement were calculated. The pulse information was used to find collection efficiencies using the measured relationships from Sec. 2.A. Finally, the ratios of calibration/measured collection efficiencies were used as correction factors for the 1000 SRS measurements.

First, the pulse dose during 1000 SRS calibration was calculated. The dose to the central chamber was calculated from the TPS. The number of pulses was calculated from the MU/min of the machine and the delivery time $(360 \mathrm{~Hz}$ when using the maximum $\mathrm{MU} / \mathrm{min}$ ).

Pulse frequency and dose during a measurement were calculated using the 1000 SRS measurement file and data from the TPS. The formula for determining the pulse dose during a measurement is shown in Eq. (1). This formula assumes a constant pulse dose throughout the individual collection intervals of the Octavius 4D, which was set to $0.2 \mathrm{~s}$ for all of the measurements. This limitation in temporal resolution makes it difficult, if not impossible to distinguish separate pulses - only the average pulse frequency during the collection interval is obtainable. As such, Eq. (1) does not calculate the actual pulse dose, rather it will calculate the average pulse dose during the $0.2 \mathrm{~s}$ collection interval.

Furthermore, the dose rate servo of the TrueBeam may modulate the pulse structures (height and width) to modulate dose rate. This correction technique assumes a change in collection efficiency due to increasing pulse frequency with increasing dose rate; however, the decrease in collection efficiency due to frequency increase may not match the decrease in collection efficiency due to a change in pulse structure. This is an inherent limitation in this correction technique, which warrants the additional investigation of a simpler correction method. 
Dose $_{\text {measured }}$ is the amount of dose measured by each chamber during a single sampling time. $\mathrm{MU} / \mathrm{min}_{\text {measured }}$ is the $\mathrm{MU} / \mathrm{min}$ of the machine during the sampling interval. $\mathrm{MU} / \mathrm{min}_{\max }$ is the maximum $\mathrm{MU} / \mathrm{min}$ of the machine for each energy: $600 \mathrm{MU} / \mathrm{min}$ for $6 \mathrm{MV}$ and $2400 \mathrm{MU} / \mathrm{min}$ for $10 \mathrm{FFF}$,

$$
\frac{\text { Dose }}{\text { Pulse }}=\frac{\text { Dose }_{\text {measured }}}{\mathrm{MU} / \mathrm{min}_{\text {measured }}} * \frac{\mathrm{MU} / \mathrm{min}_{\mathrm{max}}}{360 \text { pulses } / \mathrm{s}} * \frac{1}{\text { collection time }} \text {. }
$$

The Dose measured $_{\text {was }}$ taken directly from the Octavius 4D measurement files.

The $\mathrm{MU} / \mathrm{min}_{\text {measured }}$ was found using information from the measurement file and the TPS. The TPS stores a table containing $\mathrm{MU} / \mathrm{min}$ as a function of gantry angle. For each plan, the MU/min vs gantry angle table was exported from the TPS into the MATLAB software. The Octavius 4D system records the gantry angle for each measurement point, which was used to look up the $\mathrm{MU} / \mathrm{min}_{\text {measured }}$ from the TPS table. Planned $\mathrm{MU} / \mathrm{min}$ was used instead of the delivered MU/min due to the uncertainty in the Octavius 4D inclinometer reading that resulted in unrealistic pulse doses when measurements were matched with the delivered MU/min from the beam trajectory files. Using the planned $\mathrm{MU} / \mathrm{min}$ resulted in more realistic pulse doses, especially when there were large changes in $\mathrm{MU} / \mathrm{min}$ as a function of gantry angle.

Using the data from the previous paragraphs and Eq. (1), the pulse frequency and dose at every detector were found for each measurement point. The pulse information was used to calculate collection efficiencies via the measured relationships from Subsection 2.A. Due to the low resolution of the collection efficiency vs frequency data (Fig. 3), linear interpolation was used to calculate collection efficiencies for frequencies that were not multiples of $60 \mathrm{~Hz}$ (MU/min not multiples of 100 and $400 \mathrm{MU} / \mathrm{min}$ for $6 \mathrm{X}$ and $10 \mathrm{FFF}$, respectively). The collection efficiencies were used to determine correction factors for each measurement point. Correction factors, shown in Eq. (2), were the ratios of the collection efficiency at the time of daily calibration (CCE), divided by the collection efficiency during the measurement (MCE),

$$
\text { Correction Factor }=\frac{\mathrm{CCE}}{\mathrm{MCE}} \text {. }
$$

Each measurement point was subsequently multiplied by its corresponding correction factor.

This correction method provides corrections for changes in both pulse frequency and dose. However, the correction method is cumbersome and may not be easily implemented in standard QA practices. Therefore a second correction was tested that involved simply matching the 1000 SRS daily calibration $\mathrm{MU} / \mathrm{min}$ to the expected average $\mathrm{MU} / \mathrm{min}$ of the plan. For simplicity the daily calibration correction will be referred to as the "simple" correction, whereas the MATLAB correction will be referred to as the "complex" correction.

The simple correction only accounts for changes in pulse frequency and as such was expected to be less accurate than the complex correction for changes in pulse frequency and dose. To determine the accuracy of the simple corrections, the resulting QA pass rates were compared for both correction techniques.

One issue with the simple correction method is that the TrueBeam linear accelerator only allows the selection of discrete MU/min values during 1000 SRS calibration, making it impossible to exactly match the calibration $\mathrm{MU} / \mathrm{min}$ to that of the plan. Therefore, a clinical user may have to choose a calibration MU/min that is higher or lower than the plan. To determine whether this choice has a major effect on QA results, the effect of both high and low MU/min corrections was tested and the resulting QA pass rates were compared.

\section{E. VMAT analysis}

After applying the correction factors to the measurements, the Octavius 4D measurements were analyzed in the VeriSoft software package. VeriSoft uses PDDs to project the measured dose from the 1000 SRS throughout the phantom. Dose from each projection is summed and compared to the dose predicted by the TPS using a 3D gamma analysis. In this work, $3 \% / 3 \mathrm{~mm}$, $2 \% / 2 \mathrm{~mm}$, and $1 \% / 1 \mathrm{~mm}$ global criteria were used with a $10 \%$ threshold. In total seven patients were analyzed, with two plans per patient (6X and 10FFF), for a total of 34 arcs per energy. For each arc, an analysis was performed with and without pulse dose corrections and the differences in gamma pass rates were calculated.

To correlate the change in gamma pass rates with plan parameters, the average $\mathrm{MU} / \mathrm{min}$ and average MLC aperture size were calculated for each arc. DICOM RT plan files were imported into MATLAB, where the MLC positions and meterset weights were used to calculate the average aperture size for each arc. Planned MU/min and meterset weights taken from the DICOM files were used to calculate average $\mathrm{MU} / \mathrm{min}$. The per-arc parameters were compared to the average change in $1 \% / 1 \mathrm{~mm}$ gamma pass rate for each plan using a linear regression analysis, and Pearson correlation coefficients were calculated.

\section{RESULTS}

Figure 2 shows the collection efficiency of the 1000 SRS LICs for $6 \mathrm{MV}$ and 10FFF, averaged over four days. Uncertainties in measured collection efficiencies were less than 2.0 $\times 10^{-3}$ for both energies. Uncertainties tended to increase for lower pulse doses (higher collection efficiencies) due to lower 1000 SRS signal detection for a given MU. The results are presented without error bars for clarity; however, the uncertainty of the measurements are included in the slope uncertainties shown in Fig. 3. The collection efficiencies were linearly correlated with pulse dose. As expected, collection efficiencies increased as both pulse dose and pulse frequency decreased. The change in collection efficiencies as a function of pulse dose were slightly larger for 10FFF, changing by $5.01 \% /(\mathrm{mGy} / \mathrm{pulse})$ at $360 \mathrm{~Hz}(2400 \mathrm{MU} / \mathrm{min})$, compared to $4.44 \% /(\mathrm{mGy} /$ pulse) for $6 \mathrm{X}$, when a $360 \mathrm{~Hz}(600 \mathrm{MU} / \mathrm{min})$ pulse frequency was used.

The magnitude of the slopes from the collection efficiency versus pulse dose regression analysis is plotted against pulse 

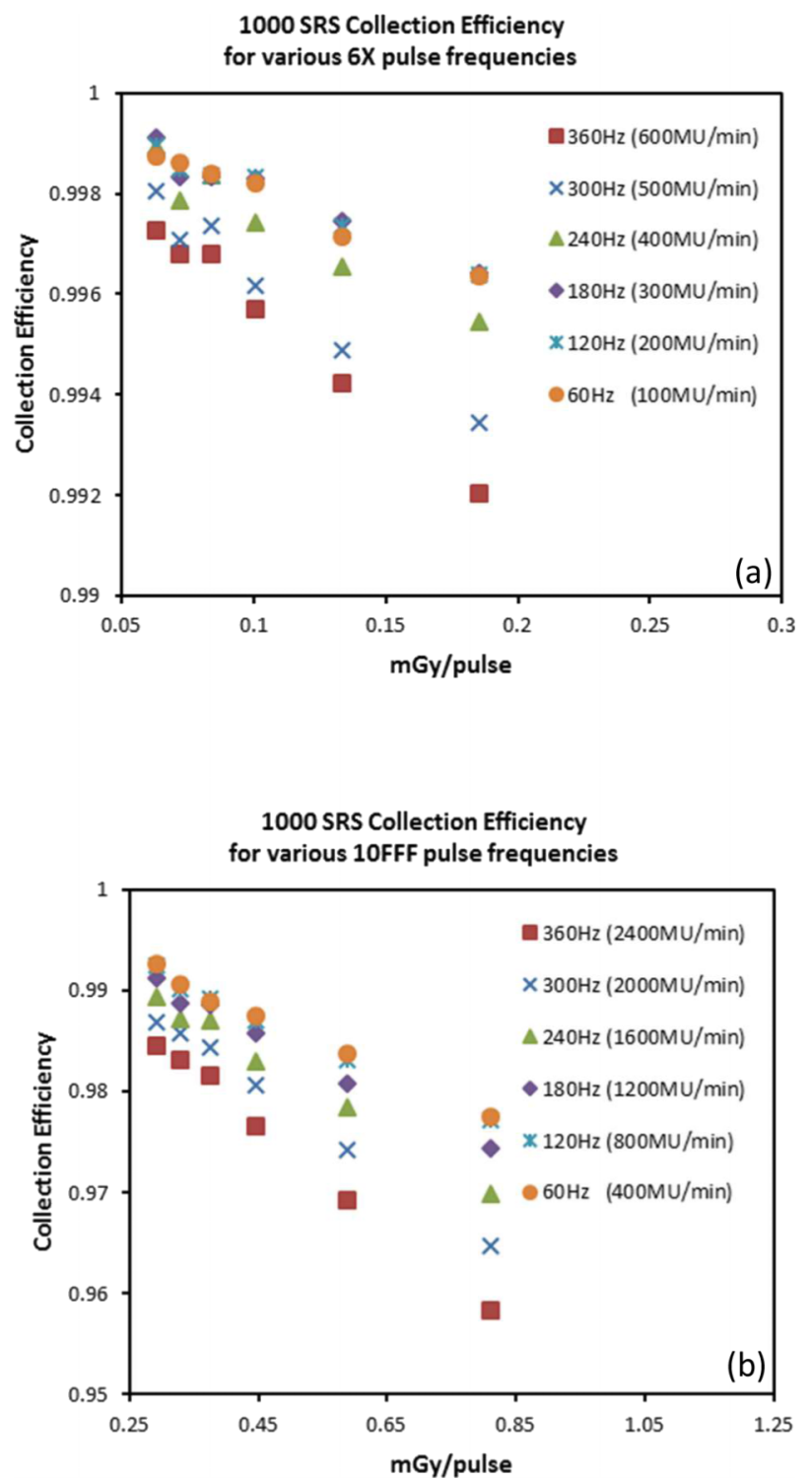

FIG. 2. 1000 SRS liquid-filled ionization chamber collection efficiencies as a function of pulse dose and pulse frequencies delivered to the detector for (a) $6 \mathrm{MV}$ and (b) 10FFF.

frequency in Fig. 3. Error bars in Fig. 3 are the calculated standard deviations of slope measurements, which were repeated over multiple days. The slope magnitudes were used in the ion recombination corrections to find the change in collection efficiencies for measurements with different combinations of pulse dose and pulse frequency.

\section{A. VMAT analysis}

The change in $3 \mathrm{D}$ gamma pass rates from the ion recombination corrections is shown in Table I for $6 \mathrm{MV}$ and 10FFF. For all fields, pass rates improved after corrections. For each plan, the changes in pass rates were averaged over all arcs. The per-plan change in pass rates, found by averaging the pass rate changes for all arcs in a single plan, was used to calculate average (Avg.), maximum (Max.), and standard deviation $(\sigma)$.

Simple corrections produced pass rates both larger and smaller than complex corrections. To quantify the difference

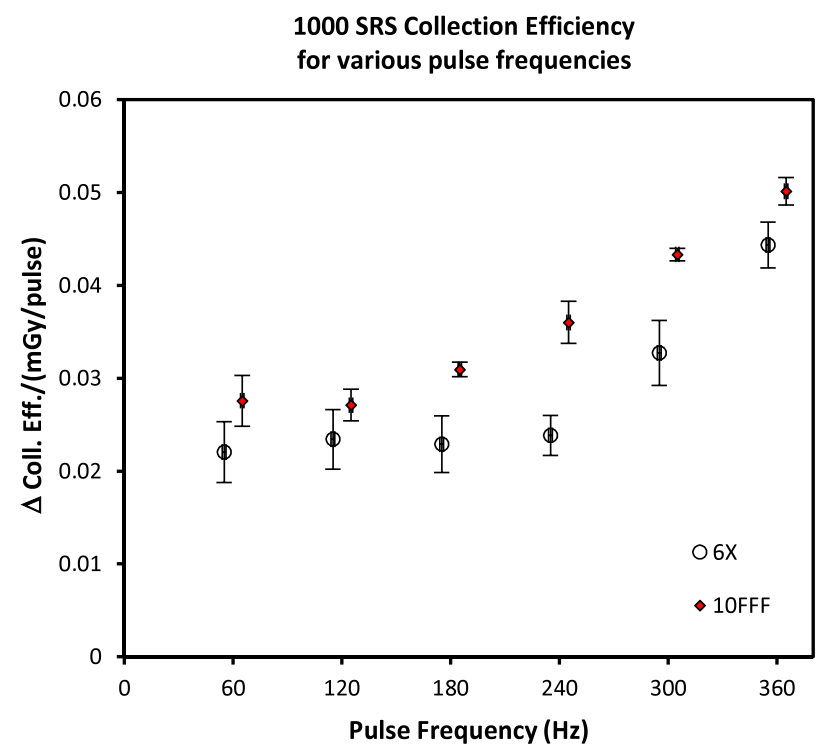

FIG. 3. Magnitude of ion recombination effects as a function of pulse frequency. All measurements are for multiples of $60 \mathrm{~Hz}(100 \mathrm{MU} / \mathrm{min}$ for $6 \mathrm{X}$ and $400 \mathrm{MU} / \mathrm{min}$ for 10FFF) — they have been offset for improved visualization. The vertical axis is the change in ion collection efficiency for a given change in pulse dose.

in pass rates between the two correction methods, the absolute difference in per-arc pass rates was calculated and the results are shown in Table II. During simple corrections the nearest possible calibration $\mathrm{MU} / \mathrm{min}$ was selected to match the plan. Both the results for rounding up (High) and rounding down (Low) to the nearest $\mathrm{MU} / \mathrm{min}$ are displayed. On average the direction of rounding had less than a $0.5 \%$ effect on pass rates. However, selection of the lower calibration MU/min produced pass rates closer to the complex corrections.

Figure 4 shows dose profiles displaying the effects of ion recombination corrections for a single arc. The dose profile is taken along the $C / A$ of the detector. The profiles are truncated below $10 \%$ of the maximum dose, to show the measurements that will be included in the gamma analysis. The uncorrected (measured) and corrected dose planes are compared to the dose predicted by the TPS. The difference between measured and corrected dose has been plotted as an absolute difference $(M$ $-C)$ and a relative difference $\left((M-C) / M^{*} 100 \%\right)$. The relative distance represents the magnitude of the ion recombination corrections at those detector locations.

Figure 5 shows the average per-plan change in pass rates due to recombination corrections plotted against average planned MLC aperture size and average MU/min. The equations from the linear regression are shown on the plots along with the Pearson correlation coefficients. Changes in pass rates were weakly correlated to average MLC aperture size; however, they tended to increase as smaller aperture sizes were used. Changes in pass rates were more strongly correlated to $\mathrm{MU} / \mathrm{min}$, increasing as average $\mathrm{MU} / \mathrm{min}$ decreased.

\section{DISCUSSION}

The linearity between collection efficiency and pulse dose, shown in Fig. 2, matches the relationships found by Chung 
TABLE I. Changes in gamma pass rates from complex ion recombination corrections. The average (Avg.), standard deviation $(\sigma)$, and maximum (Max.) changes were calculated from the per-plan changes in pass rates.

\begin{tabular}{|c|c|c|c|c|c|c|c|c|c|}
\hline \multirow[b]{3}{*}{ Energy } & \multicolumn{9}{|c|}{ 3D gamma pass rate changes } \\
\hline & \multicolumn{3}{|c|}{$3 \% / 3 \mathrm{~mm}$} & \multicolumn{3}{|c|}{$2 \% / 2 \mathrm{~mm}$} & \multicolumn{3}{|c|}{$1 \% / 1 \mathrm{~mm}$} \\
\hline & Avg. & $\sigma$ & Max. & Avg. & $\sigma$ & Max. & Avg. & $\sigma$ & Max. \\
\hline $6 \mathrm{X}$ & 0.07 & 0.16 & 0.43 & 0.40 & 0.66 & 1.63 & 1.17 & 1.14 & 3.05 \\
\hline $10 \mathrm{FFF}$ & 0.29 & 0.40 & 1.00 & 1.40 & 2.09 & 4.80 & 4.57 & 3.71 & 11.20 \\
\hline
\end{tabular}

et al. ${ }^{8}$ The magnitude of the slope of the $6 \mathrm{MV}$ linear fits was $4.44 \% /(\mathrm{mGy} / \mathrm{pulse})$ for $360 \mathrm{~Hz}$ (600 MU/min). The slope was close to the manufacturer quoted values of $3.79 \% /(\mathrm{mGy} / \mathrm{pulse})$ compared to previous publications that found slopes closer to $10.0 \% /(\mathrm{mGy} / \mathrm{pulse})$ for the $1000 \mathrm{SRS} .{ }^{14,19}$ The difference in measured collection efficiencies from previous publications may be due to the different pulse frequency $(400 \mathrm{vs} 360 \mathrm{~Hz})$ and measurement technique (two-dose rate method vs ion chamber normalization), used in the other studies.

It is important to note that the measured collection efficiencies are specific to the TrueBeam's pulse structure. When operating at the maximum dose rate, the TrueBeam delivers radiation in bundles of 6 equally spaced pulses. When a lower dose rate is used, pulses are dropped. However, the remaining pulses are not uniformly distributed in the bundles. The longer collection time in the Octavius 4D measurements, $0.2 \mathrm{~s}$ spanning 12 bundles, will provide some averaging against the pulse nonuniformity. However, the collection efficiencies shown in Fig. 2 will still be unique to the nonuniform pulse sequence of the TrueBeam and may not be applicable to other pulse structures.

The pulse structure of the TrueBeam can help explain the change in ion recombination slopes for the different pulse frequencies, shown in Figs. 2 and 3. Chung et al. described the difference in the ion recombination slopes between, pulsed and continues beams. ${ }^{8}$ The slopes of the continuous beams were steeper than the pulsed slopes. This was due to the buildup of free charge in the chamber, which will increase general recombination, resulting in a higher ion recombination effect.

Figure 3 shows the magnitude of ion recombination decreasing as pulse frequency decreases. When the TrueBeam is operating at the highest dose rate and frequency, the pulse sequence will be closest to a continuous beam. As the dose rate is lowered, pulses are dropped, reducing the buildup of free charge, resulting in a more discontinuous beam and lower slope. Uncertainties in ion recombination magnitudes were primarily due to uncertainties in (1) measuring $P_{\text {ion }}$, (2) 1000 SRS measurements, and (3) the linear regression analysis. Uncertainties in the $6 \mathrm{X}$ ion recombination magnitudes were typically larger than $10 \mathrm{FFF}$, due to the increased collection of leakage in longer (lower MU/min) 1000 SRS measurements.

\section{A. VMAT analysis}

The effects of ion recombination corrections on pass rates were larger for 10FFF compared to $6 \mathrm{X}$. The larger changes in pass rates were caused by three differences: (1) the pulse dose at the central axis of the 1000 SRS in Octavius 4D was larger for 10FFF ( $0.760 \mathrm{mGy} /$ pulse) compared to $6 \mathrm{MV}$ (0.175 mGy/pulse), (2) as seen in Fig. 3, a change in pulse dose results in a larger change in collection efficiency for 10FFF, and (3) as seen in Fig. 5, the average MU/min of 10FFF plans deviated further from calibration ( $\max \mathrm{MU} / \mathrm{min}$ ), compared to $6 \mathrm{MV}$. Changes in pass rates, due to ion recombination corrections, increased with tighter gamma analysis criteria. Tighter gamma criteria have been recommended for SBRT QA ( $2 \% / 1 \mathrm{~mm}$ or less), due to the high conformity, small field size, and hypofractionation of SBRT treatments. ${ }^{20,21}$ Therefore, the tighter gamma criteria, where ion recombination corrections are the largest, will likely be used in a clinical analysis.

Ion recombination corrections tend to have a uniform effect on the VMAT dose distribution. As seen in Fig. 4, the magnitude of the ion recombination effect $\left((M-C) / M^{*} 100 \%\right)$ is mostly uniform in the high dose region ( $>10 \%$ max dose). There is some nonuniformity in the high dose regions near the high dose gradients of the profiles. During a VMAT delivery, the high dose gradients are created by exposing a MLC edge at that location, which results in a dosimetric penumbra with a

TABLE II. Comparison of the 3D gamma pass rate results obtained after simple and complex ion recombination corrections. The average (Avg.), standard deviation $(\sigma)$, and maximum (Max.) changes were calculated from the absolute values of per-arc changes in pass rates.

\begin{tabular}{|c|c|c|c|c|c|c|c|c|c|c|}
\hline \multirow[b]{3}{*}{ Energy } & \multirow[b]{3}{*}{$\mathrm{MU} / \mathrm{min}$} & \multicolumn{9}{|c|}{ Pass rate difference between correction methods } \\
\hline & & \multicolumn{3}{|c|}{$3 \% / 3 \mathrm{~mm}$} & \multicolumn{3}{|c|}{$2 \% / 2 \mathrm{~mm}$} & \multicolumn{3}{|c|}{$1 \% / 1 \mathrm{~mm}$} \\
\hline & & Avg. & $\sigma$ & Max. & Avg. & $\sigma$ & Max. & Avg. & $\sigma$ & Max. \\
\hline \multirow{2}{*}{$6 \mathrm{X}$} & High & 0.03 & 0.05 & 0.1 & 0.21 & 0.19 & 0.5 & 0.78 & 0.30 & 1.2 \\
\hline & Low & 0.01 & 0.02 & 0.0 & 0.10 & 0.06 & 0.2 & 0.43 & 0.28 & 0.7 \\
\hline \multirow{2}{*}{$10 \mathrm{FFF}$} & High & 0.01 & 0.02 & 0.0 & 0.27 & 0.16 & 0.5 & 0.98 & 0.58 & 2.2 \\
\hline & Low & 0.00 & 0.01 & 0.0 & 0.18 & 0.08 & 0.3 & 0.80 & 0.42 & 1.7 \\
\hline
\end{tabular}



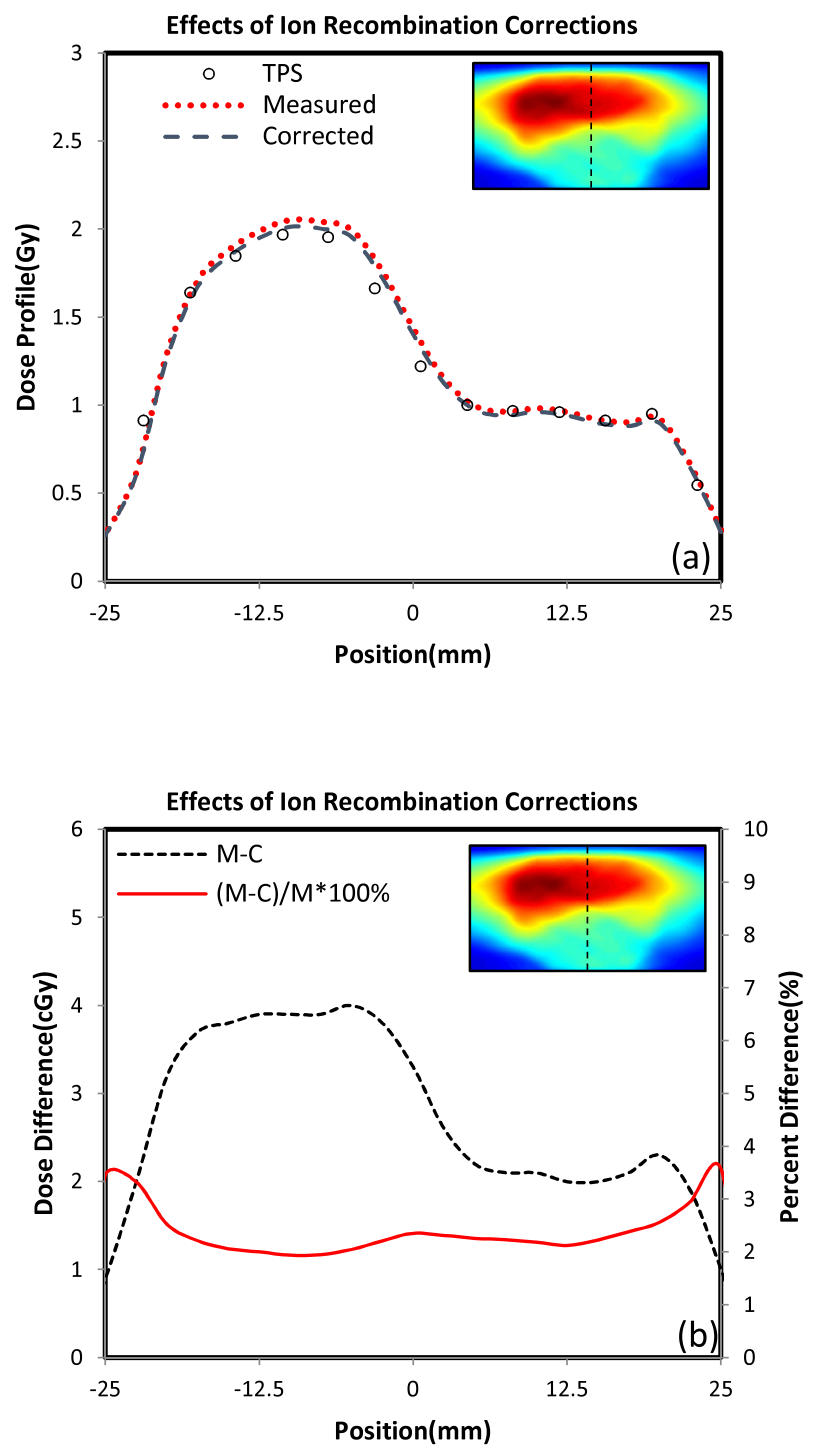

FIG. 4. Dosimetric effects of complex ion recombination corrections in the coronal plane of the Octavius 4D phantom. (a) Measured and ion recombination corrected dose profiles along the central axis of the 1000 SRS compared to the TPS predicted dose. (b) Difference between measured and corrected profiles. The relative distance is plotted on the right vertical axis.

spatially varying pulse dose. The change in pulse dose from the calibration conditions is the cause of the increased ion recombination effects at these locations.

Although there is some nonuniformity in ion recombination effects across a VMAT field, the effect tends to be uniform in the high dose region ( $>10 \%$ max dose) - the region that will be included in the VMAT analysis. Across the subset of LICs measuring in the high-dose region of a VMAT distribution, ion recombination corrections tend to be uniform within the subset and dependent upon rep-rate only due to the majority of the dose being delivered to these chambers directly through open MLC apertures at similar pulse doses. The uniformity of ion recombination effects is expected to be similar for static-gantry IMRT and nonSBRT deliveries, given similar conditions of uniform pulse doses within the high dose region of the field. This uniformity of the ion recombination effect is promising, in that it may be possible to apply a single calibration factor,
Dose-per-pulse Corrections vs Aperture Size

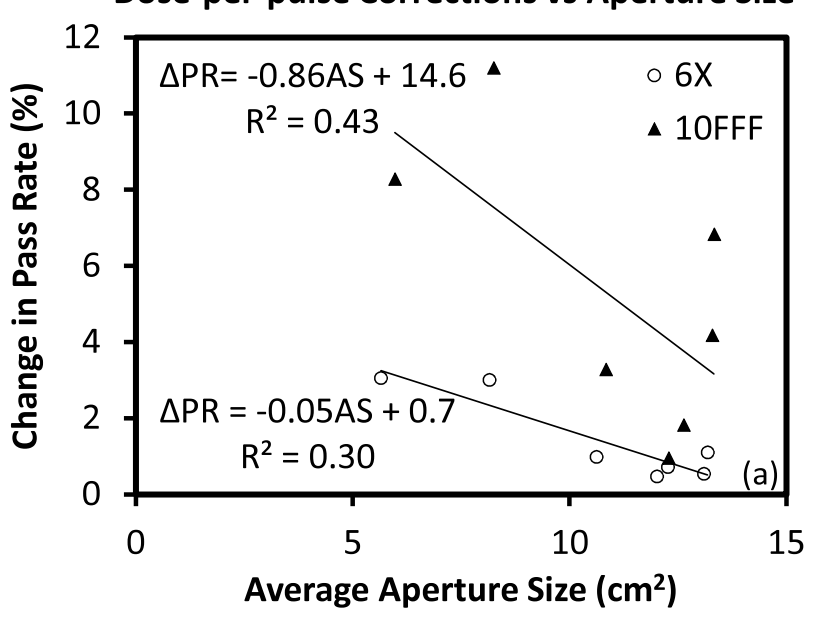

Dose-per-pulse Corrections vs MU/min

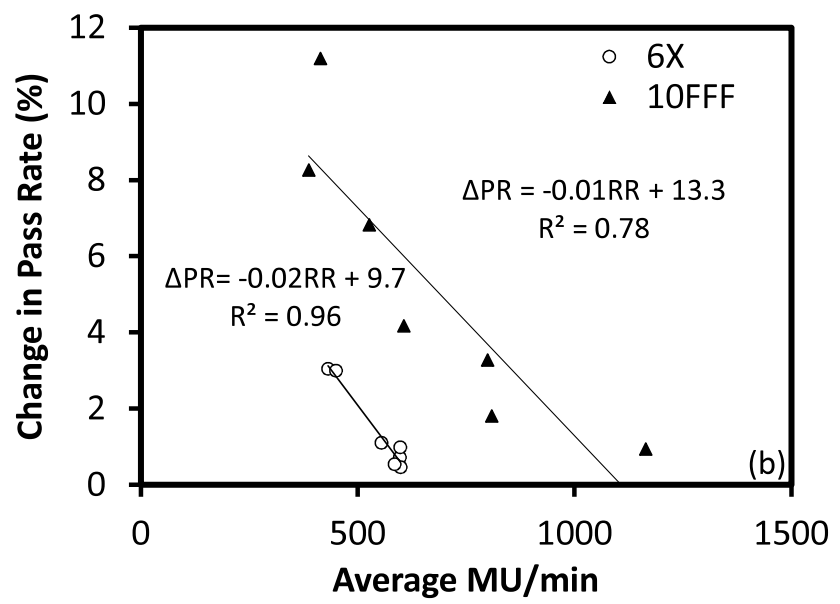

FIG. 5. Correlation between changes in 3D gamma analysis pass rates ( $\triangle \mathrm{PRs})$ from pulse dose corrections and (a) average MLC aperture size (AS), (b) average $\mathrm{MU} / \mathrm{min}$ (RR). 3D gamma analysis was performed using a $1 \% / 1 \mathrm{~mm}$ criteria with a $10 \%$ dose threshold.

which could account for this effect. The daily calibration of the 1000 SRS presents itself as a natural opportunity for the application of a global calibration factor to account for ion recombination.

The inclusion of an ion recombination correction in the daily calibrations requires an identification of the magnitude of recombination prior to delivery. For this reason, the magnitudes of the ion recombination effects were correlated to average planned $\mathrm{MU} / \mathrm{min}$ and aperture size-parameters that will change pulse frequency and pulse dose, respectively. Figure 5 shows the results of this investigation, where it was found that the magnitudes of ion recombination effects are more strongly correlated to $\mathrm{MU} / \mathrm{min}$.

The stronger correlation suggests that the magnitudes of the ion recombination effects in the 1000 SRS measurements are larger for changes in MU/min, rather than MLC aperture size. The reasoning for the increased effect can be discerned from the measurement and the process used to calibrate the 1000 SRS. The magnitudes of the dosimetric errors due to the ion recombination effect at any point in the measurement are 
equal to the change in collection efficiency multiplied by the dose per pulse. The 1000 SRS was calibrated at the maximum pulse dose and pulse frequency. Lowering the pulse frequency (MU/min) will change the collection efficiency, while keeping pulse dose larger, resulting in a large ion recombination effect. Lowering the pulse dose (MLC aperture size) will change the collection efficiency; however, there will be a smaller dosimetric error compared with the change in frequency. It is this characteristic of the ion recombination effects that results in larger dosimetric errors for changing frequency (MU/min).

Fortunately, ion recombination corrections for changing $\mathrm{MU} / \mathrm{min}$ can easily be included in the daily calibration by matching the calibration $\mathrm{MU} / \mathrm{min}$ to the average $\mathrm{MU} / \mathrm{min}$ of the plan. This was implemented in the simple ion recombination corrections. From Table II it can be seen that the simple corrections greatly reduce errors due to ion recombination effects. There is still some difference in pass rates between the complex and simple corrections. This is due to the residual change in $\mathrm{MU} / \mathrm{min}$ and aperture size during the VMAT delivery. It may be possible to match the average aperture size during calibration to that of delivery. However, the average aperture size of the plan is not readily available from the treatment planning system. The added complexity of calculating aperture size may be an unrealistic burden for clinics looking for a simple fix to ion recombination effects. Furthermore, as MLC aperture sizes increase and more closely resemble calibration conditions, ion recombination effects will become more dominated by changes in $\mathrm{MU} / \mathrm{min}$. As such, it is expected that simple corrections for MU/min will more closely approximate complex corrections.

Choosing a calibration $\mathrm{MU} / \mathrm{min}$ higher or lower than the planned $\mathrm{MU} / \mathrm{min}$ produced pass rates within $0.5 \%$. On average the lower $\mathrm{MU} / \mathrm{min}$ calibration pass rates were closer to the complex corrections. This is likely due to the increased collection efficiency during calibration from the lower MU/min accounting for the increased collection efficiency from the lower aperture size.

Many clinics use a $6 \mathrm{MV}$ flattening filter free mode (6FFF) to treat SBRT patients. The removal of the flattening filter for 6FFF more than doubles the maximum pulse dose of the machine. The pulse dose at the central axis of the 1000 SRS in the Octavius 4D during calibration would increase from $0.175 \mathrm{mGy} /$ pulse to approximately $0.406 \mathrm{mGy} /$ pulse, which is less than the $0.760 \mathrm{mGy} /$ pulse of the 10FFF beam. The result is that errors in pass rate due to ion recombination effects would likely fall somewhere between the $6 \mathrm{X}$ and 10FFF results. However, the residual error in pass rates after the simple corrections, shown in Table II, was similar for $6 \mathrm{X}$ and 10FFF. Therefore, the residual error in 6FFF measurements would be expected to follow the same trend.

The discussion of the ion recombination effects relies on the accuracy of the ion recombination corrections, which use measured pulse dose and pulse frequency to calculate collection efficiencies. However, there is an error introduced into the pulse dose corrections when calculating the measurement collection efficiencies. The true pulse dose is needed to determine accurate collection efficiencies. However, only the measured pulse dose, which is effected by the collection efficiency, is available leading to an inherent error in the collection efficiency calculation. The magnitude of this effect can be determined by (1) assuming a true pulse dose, (2) calculating the corresponding measured pulse dose, (3) using Eq. (2) to recalculate true pulse dose from the measured, and (4) finally comparing the result to the original assumed true pulse dose. Using this technique, the error in calculating dose from measured instead of true pulse dose in the ion recombination corrections is less than $0.1 \%$ for all energies.

\section{CONCLUSION}

1000 SRS collection efficiencies increased with decreasing pulse dose and pulse frequency. For a given pulse dose and a $360 \mathrm{~Hz}$ pulse frequency (600 and $2400 \mathrm{MU} / \mathrm{min}$ for $6 \mathrm{X}$ and 10FFF, respectively), the ion recombination was $4.44 \% /(\mathrm{mGy} /$ pulse) and $5.01 \% /(\mathrm{mGy} /$ pulse) for $6 \mathrm{MV}$ and 10FFF, respectively. On average, applying complex pulse dose and pulse frequency corrections to 1000 SRS measurements produced small changes in $6 \mathrm{X} 3 \mathrm{D}$ gamma pass rates $(1.17 \% \pm 1.14 \%$ for $1 \% / 1 \mathrm{~mm}$ gamma criteria). However, the same corrections resulted in larger changes in pass rates for $10 \mathrm{FFF}(4.57 \% \pm 3.71 \%$ for $1 \% / 1 \mathrm{~mm}$ gamma criteria $)$, which increased when the plan contained small aperture sizes and strict gamma criteria were used. The magnitudes of the change in pass rates were strongly correlated to pulse frequency $\left(r^{2}=0.96\right.$ for $6 \mathrm{X}$ and $r^{2}=0.78$ for 10FFF); therefore, a simple correction method was tested for which the MU/min of the 1000 SRS calibration was selected to match the average planned VMAT MU/min. On average the pass rates of the simple corrections were within $1 \%$ of the complex correction pass rates for all energies and gamma criteria.

\section{ACKNOWLEDGMENT}

This research was supported by PTW (Freiburg, Germany).

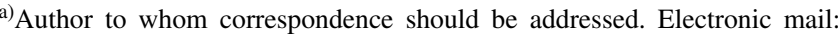
knillcor@gmail.com; Telephone: (313) 576-9646.

${ }^{1}$ A. Gago-Arias, L. Brualla-Gonzalez, D. M. Gonzalez-Castano, F. Gomez, M. S. Garcia, V. L. Vega, J. M. Sueiro, and J. Pardo-Montero, "Evaluation of chamber response function influence on IMRT verification using 2D commercial detector arrays," Phys. Med. Biol. 57(7), 2005-2020 (2012).

${ }^{2}$ Z. Allibhai, M. Taremi, A. Bezjak, A. Brade, A. J. Hope, A. Sun, and B. C. Cho, "The impact of tumor size on outcomes after stereotactic body radiation therapy for medically inoperable early-stage non-small cell lung cancer," Int. J. Radiat. Oncol., Biol., Phys. 87(5), 1064-1070 (2013).

${ }^{3}$ I. J. Das, G. X. Ding, and A. Ahnesjo, "Small fields: Nonequilibrium radiation dosimetry," Med. Phys. 35(1), 206-215 (2008).

${ }^{4} \mathrm{~W}$. U. Laub and T. Wong, "The volume effect of detectors in the dosimetry of small fields used in IMRT," Med. Phys. 30(3), 341-347 (2003).

${ }^{5} \mathrm{G}$. Wickman and H. Nystrom, "The use of liquids in ionization chambers for high-precision radiotherapy dosimetry," Phys. Med. Biol. 37(9), 1789-1812 (1992).

${ }^{6}$ K. J. Stewart, A. Elliott, and J. P. Seuntjens, "Development of a guarded liquid ionization chamber for clinical dosimetry," Phys. Med. Biol. 52(11), 3089-3104 (2007).

${ }^{7}$ B. Johansson, G. Wickman, and J. Bahar-Gogani, "General collection efficiency for liquid isooctane and tetramethylsilane in pulsed radiation," Phys. Med. Biol. 42(10), 1929-1938 (1997). 
${ }^{8}$ E. Chung, S. Davis, and J. Seuntjens, "Experimental analysis of general ion recombination in a liquid-filled ionization chamber in high-energy photon beams," Med. Phys. 40(6), 062104 (7pp.) (2013).

${ }^{9}$ E. Johansson, J. Andersson, L. Johansson, and H. Tolli, "Liquid ionization chamber initial recombination dependence on LET for electrons and photons," Phys. Med. Biol. 58(12), 4225-4236 (2013).

${ }^{10} \mathrm{~J}$. Pardo-Montero and F. Gomez, "Determining charge collection efficiency in parallel-plate liquid ionization chambers," Phys. Med. Biol. 54(12), 3677-3689 (2009).

${ }^{11} \mathrm{H}$. Tolli, R. Sjogren, and M. Wendelsten, "A two-dose-rate method for general recombination correction for liquid ionization chambers in pulsed beams," Phys. Med. Biol. 55(15), 4247-4260 (2010).

${ }^{12}$ J. Andersson, E. Johansson, and H. Tolli, "On the property of measurements with the PTW microLion chamber in continuous beams," Med. Phys. 39(8), 4775-4787 (2012).

${ }^{13}$ C. Martens, C. De Wagter, and W. De Neve, "The value of the LA48 linear ion chamber array for characterization of intensity-modulated beams," Phys. Med. Biol. 46(4), 1131-1148 (2001).

${ }^{14}$ B. Poppe, T. S. Stelljes, H. K. Looe, N. Chofor, D. Harder, and K. Willborn, "Performance parameters of a liquid filled ionization chamber array," Med. Phys. 40(8), 082106 (14pp.) (2013).

${ }^{15}$ C. K. McGarry, B. F. O'Connell, M. W. Grattan, C. E. Agnew, D. M. Irvine, and A. R. Hounsell, "Octavius 4D characterization for flattened and flattening filter free rotational deliveries,” Med. Phys. 40(9), 091707 (11pp.) (2013).
${ }^{16}$ P. R. Almond, P. J. Biggs, B. M. Coursey, W. F. Hanson, M. S. Huq, R. Nath, and D. W. Rogers, "AAPM's TG-51 protocol for clinical reference dosimetry of high-energy photon and electron beams," Med. Phys. 26(9), 1847-1870 (1999).

${ }^{17}$ D. A. Low, W. B. Harms, S. Mutic, and J. A. Purdy, "A technique for the quantitative evaluation of dose distributions," Med. Phys. 25(5), 656-661 (1998).

${ }^{18}$ K. B. Pulliam, J. Y. Huang, R. M. Howell, D. Followill, R. Bosca, J. O'Daniel, and S. F. Kry, "Comparison of 2D and 3D gamma analyses," Med. Phys. 41(2), 021710 (6pp.) (2014).

${ }^{19}$ M. Markovic, S. Stathakis, P. Mavroidis, I. A. Jurkovic, and N. Papanikolaou, "Characterization of a two-dimensional liquid-filled ion chamber detector array used for verification of the treatments in radiotherapy," Med. Phys. 41(5), 051704 (14pp.) (2014).

${ }^{20}$ S. H. Benedict, K. M. Yenice, D. Followill, J. M. Galvin, W. Hinson, B. Kavanagh, P. Keall, M. Lovelock, S. Meeks, L. Papiez, T. Purdie, R. Sadagopan, M. C. Schell, B. Salter, D. J. Schlesinger, A. S. Shiu, T. Solberg, D. Y. Song, V. Stieber, R. Timmerman, W. A. Tome, D. Verellen, L. Wang, and F. F. Yin, "Stereotactic body radiation therapy: The report of AAPM Task Group 101," Med. Phys. 37(8), 4078-4101 (2010).

${ }^{21}$ J. I. Kim, S. Y. Park, H. J. Kim, J. H. Kim, S. J. Ye, and J. M. Park, "The sensitivity of gamma-index method to the positioning errors of highdefinition MLC in patient-specific VMAT QA for SBRT," Radiat. Oncol. 9, 167-179 (2014). 